\title{
Performance Analysis of various Codecs using RSVP on VolP Quality of Service over Variable Bandwidth
}

\author{
B. K. Mishra \\ Principal, Thakur College of \\ Engineering and Technology \\ Thakur Village, \\ Kandivali (East) \\ Mumbai-400101
}

\author{
S. K. Singh \\ Research Scholar-Mukesh \\ Patel School of Technology \\ Management and \\ Engineering \\ NMIMS University, Santacruz \\ Mumbai-400056
}

\author{
Kalpana Patel \\ PG Student -Thakur College of \\ Science and Commerce, \\ Thakur Village, \\ Kandivali (East) \\ Mumbai -400101
}

\begin{abstract}
With the rapid growth of the Internet, customers are demanding multimedia applications such as telephony and video on demand, to be available on the internet. Voice over Internet Protocol (VoIP) is a rapidly emerging technology for voice communication that uses the ubiquity of IP-based networks. The greatest challenge is the provisioning of Quality of Service (QoS) over limited bandwidth. Multimedia applications need a lot more bandwidth and have different QoS requirements than the applications that were used in early years of the internet. The purpose of this paper is to check the performance of VoIP application under different Codec's such as G.711, G.729 and G.723.1 over variable bandwidth. This paper proposes RSVP protocol for providing end to end QoS for real time applications over diverse networks. The performance is checked under RSVP and Non RSVP. Packet end to end delay and packet delay variation are evaluated through simulation. The result shows that under any bandwidth G.723.1 gives better result as compared to G.711 and G.729. Simulation is done using OPNET IT Guru Academic edition.
\end{abstract}

\section{General Terms}

Performance, Design, Verification.

\section{Keywords}

QoS-Quality of service, CODEC, RSVP- Reservation Protocol, VoIP - Voice over Internet Protocol

\section{INTRODUCTION}

The keen interest in Voice over Internet Protocol (VoIP) and Internet Protocol (IP) Telephony and its' accelerated adoption rate has led many to the realization that the long-established Public Switched Telephone Network (PSTN) model will be replaced in the next 10 to 15 years. Voice over Internet Telephony (VoIP) is becoming the preferred choice of deployment all over the world. Telecommunication companies around the globe are embarking in VoIP. As more and more users are using the VoIP network, Quality of Services (QoS) will become a major area that need to be addressed at. This is to ensure the reliability, availability and quality of the calls is as good as the PSTN network. VoIP technology uses Internet Protocol (IP) network to transmit voice. Voice is packetised and sends over the IP network to the destination [1].
Generally PSTN are routed on circuit switched network whereas VoIP are routed via packet switched network. Currently, VoIP is one of the fastest growing technologies in the world. However, VoIP services are susceptible to IP network burstiness causing packets drop and momentary gap in the call. Because of the bursty nature of digital communications traffic, even the most over provisioned network will have interruption [16]. The quality of the VoIP calls is also very much dependent on factors such as latency and jitter. Latency occurs when data are delivered too slowly, usually due to congestion and jitter is a variation of packet delays. Latency and jitter can cause packet drop.

Resource Reservation Protocol (RSVP) is a proposed IETF standard for requesting and negotiating resource allocations between end users and networks or among network components. RSVP is broadly accepted in different QOS mechanisms. The main objective of this paper is to study the effect of various voice codec schemes on the VoIP Quality of Service (QoS) using RSVP protocol over variable bandwidth. Popular voice codecs such as G.711, G.729a, G.723.1 and G.726 are studied and VoIP simulation models are built. The voice codec performance is determined with the best performance codec is identified through current research work.

\section{RELATED WORK}

The term Voice over Internet Protocol, or VoIP, has been used as a catch-all phrase in the industry to refer collectively to a large group of technologies designed to provide Internet-based communications services [11]. More accurately, VoIP refers only to the underlying transport protocol that encapsulates voice traffic or voice media streams and allows them to be carried over data networks, using IP network technologies or internet protocols. VoIP, however, is not IP Telephony, nor is it the more widely used industry terminology as IP communications that refers to an even broader definition of communications applications.

As a signaling protocol, RSVP has different functionalities in different QoS mechanisms. In IntServ, RSVP is used for specifying resource requirements of real-time flows. A sender that wishes to initiate a session send a PATH message to the corresponding receiver, containing traffic parameters and QoS requirement of the sending application. The receiver then generates a RESV message to request the resources in each node along the path. The intermediate nodes may accept or reject the 
request when receiving RESV. If the sender successfully receives the RESV message, meaning that the end-to-end resources have been reserved for the flow, the sender starts to transfer data. In DiffServ, the Expedited Forwarding (EF) service is suitable for Internet Telephony and videoconferencing [3] [20]. However, EF services are expensive. In order to meet requirements for Next generation Network, some proposal to support on mobility as MRSVP [19] has been discussed in past. RSVP Flow Transparency and Localized RSVP attempted to resolve mobility issues with RSVP. However, due to the complexity and difficulty in supporting large signaling messages, mobility, congestion control and security, these approaches which were directly derived from RSVP failed to achieve wide acceptance [2][15]. Few efforts has been taken in past to discuss performance analysis by using various codecs but as such does not achieve wide acceptance [18].

\section{DIFFERENT VOICE CODECS}

Voice transmission is analogical, whereas the data network is digital. The process to sample analogical waves into digital information is made by an encoder-decoder (CODEC). There are many standards to sample an analogical voice signal into a digital one. The process is often quite complex. Most of the conversions use pulse code modulation (PCM) or variations. The bandwidth is used more efficiently with the application of voice compression codec. The compression of the waveform can save bandwidth. This is especially interesting in low speed connections so one can have more VoIP connections at the same time. Another way to save bandwidth is using the silence suppression. Voice codec is used to compress and decompress analogue voice signal into digital format for transmitting in the packet network. During call setup, voice terminal or gateways can automatically negotiate on which codec to be used from the codec selection list that these equipments support.

The popular voice codecs used in the telecommunication industry are G.711 which is widely used in the PSTN environment [5][6], G.729 which is using Conjugate-Structure code modulation (PCM) channel to and from a 40, 32, 24 or 16 Algebraic-CodeExcited Linear-Prediction (CS-ACELP) algorithm [7], G.726 which can be used to convert a $64 \mathrm{kbit} / \mathrm{s}$ A-law or $\mu$-law pulse kbit/s channel [8], and G.723.1 which has two-bit rates associated with it, 5.3 and $6.3 \mathrm{kbit} / \mathrm{s}$ [9]. There are also some other codec like G.113 which reflects transmission impairments due to speech processing recognized by ITU [10].

\section{MAINTAINING QUALITY OF SERVICE}

In multimedia based services QoS is essentially having two phases. Initial setup phase and real-time multimedia exchange phases. In the audio based application, end to end transmission delay should be small enough so that interference should not affect the normal conversation [12]. Resource Reservation Protocol (RSVP) do resource negotiation between end users and network components to make sure that enough transport resources are provided to deliver multimedia flows promptly. In the generic Internet based solutions, call signaling and resource negotiation are handled by separate protocols. A number of studies based on the integration of call signaling with resource negotiation and maintaining quality of service over next generation network can be found in the literature [15][16][22].

VoIP QoS is determined by many factors and the most important parameters are the voice codec used for the VoIP call, the one way end-to-end delay of the voice packet and packet delay variations or jitter. Voice codecs using higher compression will degrade the QoS much more than the lower compression codecs. The higher compression codec will introduce additional delay due to longer processing time at the gateway or IP phones but gives higher bandwidth efficiency. ITU-T Recommendation G.114 recommends the following limits for one-way end-to-end transmission time [5].

- 0 to $150 \mathrm{~ms}$ : Acceptable for most user applications.

- 150 to $400 \mathrm{~ms}$ : Acceptable provided that Administrations are aware of the transmission time impact on the transmission quality of user applications.

- Above $400 \mathrm{~ms}$ : Unacceptable for general network planning purpose. However, it is recognized that in some exceptional cases this limit will be exceeded.

The main problems of a VoIP quality of service are latency, jitter, packet loss and echo. Therefore it is important to design the network running VoIP application not to exceed $150 \mathrm{~ms}$ one-way delay or the QoS will be severely affected. Jitter is the variation of delay the voice packet experience when transversing across the network. Now a day most of the VoIP networks deployed come with dynamic jitter buffer to minimize the jitter effect on the VoIP QoS. The jitter buffer is responsible to re-ordered out of sequence packets and to remove duplicate packets. Packets that arrive much later than the allocated jitter buffer period will be discarded. The choice of voice codec and the one-way delay the voice packet experienced will determine the quality of the VoIP calls. Jitter and silence suppression technique implemented will also effect the VoIP quality of service.

In order to provide a requested QoS, the nodes of a network must perform session initiation phase, reservation setup, admission control, policy control, packet scheduling, and packet classification functions.

In the current research work reservation setup protocol is used to pass the QoS request originating in an end-system to each router along the data path, or in the case of multicasting, to each router along the branches of the delivery tree. In particular, it enables QoS more effective and there is considerable amount of reduction in packet end to end delay and packet delay variation which is reflecting in results. RSVP can be also used for the reservation setup protocol for heterogeneous network and it has been extensively discussed in past [13] [14].

\section{NETWORK DESIGN AND CONFIGURATION FOR RSVP}

The following network design has been taken into consideration. At the first step single traffic is used for each of the functions such as Ftp, Video Conferencing and VoIP which is shown in Fig. 1. For $3 \mathrm{G}$ and $4 \mathrm{G}$ requirement the basic architecture can be modified with reference of Bandwidth. A reference bandwidth of $50 \mathrm{Mbps}$ to $100 \mathrm{Mbps}$ is considered for $3 \mathrm{G}$ network and tested multimedia content delivery over this network.

For $4 \mathrm{G}$ networks the architecture has been modified and a reference bandwidth up to $1 \mathrm{Gbps}$ is considered and tested multimedia content delivery over this network. In both case we have measured end to end delay and packet delay variation. The below configurations applied in the Opnet and simulated to get results. 


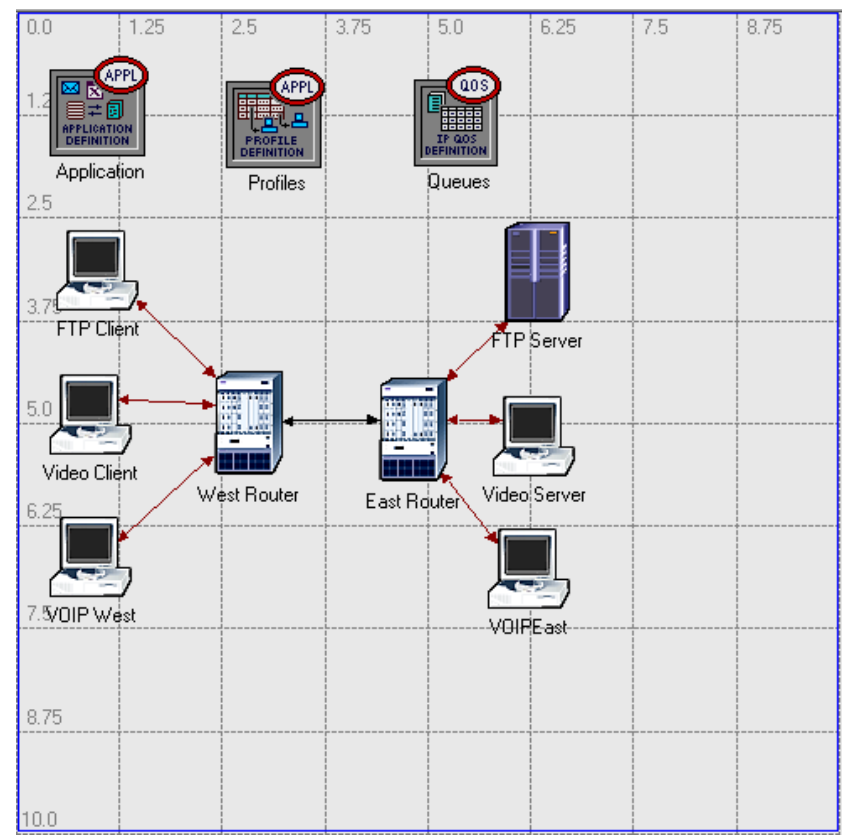

Fig 1. Network Architecture for RSVP

\section{SIMULATIONS RESULTS AND ANALYSIS}

Simulation has been done using OPNET software for variable bandwidth condition. Here the evaluation has been done for $10 \mathrm{Mbps}, 100 \mathrm{Mbps}$ and $1 \mathrm{Gbps}$ which are the expected bandwidth of $3 \mathrm{G}$ and $4 \mathrm{G}$ networks. Packet end to end delay and packet delay variation is measured.

It is tested for voice traffic under different CODECS like G.711, G.723.1, and G.729. Results have been compared with and without RSVP.

From Table 1 to Table 6, show the detailed analysis of packet end to end delay and Packet delay variation in seconds for voice traffic with different CODECS over variable bandwidth. Results have been taken with and without RSVP. Results shows, packet end to end delay is comparatively less in RSVP. It also shows the detail analysis of packet delay variation in seconds for voice traffic over variable bandwidth. Results show that packet delay variation is very less in RSVP. It also shows that, CODEC G.723.1 gives better performance as compared to other codecs when used with RSVP.A detailed comparative analysis has been done by considering variable bandwidth condition and packet delay variation and packet end to end delay is plot which is shown from fig 2 to Fig 19.

TABLE 1. STATISTICS OF PACKET END TO END DELAY FOR VOIP OVER VARIABLE BANDWIDTH WITH CODEC G.711

\begin{tabular}{|c|c|c|c|}
\hline BANDWIDTH & MAXIMUM \\
& $\begin{array}{c}\text { RESERVABLE } \\
\text { BANDWIDTH }\end{array}$ & \multicolumn{2}{|c|}{$\begin{array}{c}\text { PACKET END TO END DELAY } \\
\text { (SEC) }\end{array}$} \\
\cline { 3 - 4 } & & NON & RSVP \\
\hline \multirow{2}{*}{$10 \mathrm{MBPS}$} & $35 \%$ & 0.124042 & 0.0096688742 \\
\cline { 2 - 4 } & $50 \%$ & 0.12405 & 0.0079470199 \\
\cline { 2 - 4 } & $75 \%$ & 0.159138 & 0.0058609272 \\
\hline
\end{tabular}

\begin{tabular}{|c|c|c|c|}
\hline \multirow{3}{*}{$100 \mathrm{MBPS}$} & $35 \%$ & 0.0890863 & 0.0078145695 \\
\cline { 2 - 4 } & $50 \%$ & 0.089104 & 0.0068874172 \\
\cline { 2 - 4 } & $75 \%$ & 0.160737 & 0.0060927152 \\
\hline \multirow{3}{*}{1 GBPS } & $35 \%$ & 0.139037 & 0.0090066225 \\
\cline { 2 - 4 } & $50 \%$ & 0.139055 & 0.0078145695 \\
\cline { 2 - 4 } & $75 \%$ & 0.166396 & 0.0062251656 \\
\hline
\end{tabular}

TABLE 2. STATISTICS OF PACKET DELAY VARIATION OVER VARIABLE BANDWIDTH WITH CODEC G.711

\begin{tabular}{|l|c|c|c|}
\hline BANDWIDTH & $\begin{array}{c}\text { MAXIMUM } \\
\text { RESERVABLE } \\
\text { BANDWIDTH }\end{array}$ & \multicolumn{2}{|c|}{$\begin{array}{c}\text { PACKET DELY VARIATION } \\
\text { (SEC) }\end{array}$} \\
\cline { 3 - 4 } & & NON RSVP & RSVP \\
\hline \multirow{3}{*}{$10 \mathrm{MBPS}$} & $35 \%$ & 0.00107407 & 0.000327814 \\
\cline { 2 - 4 } & $50 \%$ & 0.00107592 & 0.000288079 \\
\cline { 2 - 4 } & $75 \%$ & 0.00229768 & 0.000197019 \\
\hline \multirow{3}{*}{ 100MBPS } & $35 \%$ & 0.00070055 & 0.000327814 \\
\cline { 2 - 4 } & $50 \%$ & 0.00070190 & 0.000291390 \\
\cline { 2 - 4 } & $75 \%$ & 0.00238222 & 0.000251655 \\
\hline 1 GBPS & $35 \%$ & 0.00148132 & 0.000339404 \\
\cline { 2 - 4 } & $50 \%$ & 0.00148153 & 0.000301324 \\
\cline { 2 - 4 } & $75 \%$ & 0.00256809 & 0.000230132 \\
\hline
\end{tabular}

TABLE 3. STATISTICS OF PACKET END TO END DELAY FOR VOIP OVER VARIABLE BANDWIDTH WITH CODEC G.723.1

\begin{tabular}{|c|c|c|c|}
\hline BANDWIDTH & $\begin{array}{c}\text { MAXIMUM } \\
\text { RESERVABLE }\end{array}$ & \multicolumn{2}{|c|}{$\begin{array}{c}\text { PACKET END TO END } \\
\text { DELAY (SEC) }\end{array}$} \\
\cline { 3 - 4 } & BANDWIDTH & $\begin{array}{c}\text { NON } \\
\text { RSVP }\end{array}$ & RSVP \\
\hline \multirow{2}{*}{10 MBPS } & $35 \%$ & 0.0449134 & 0.0052251656 \\
\cline { 2 - 4 } & $50 \%$ & 0.0449156 & 0.0046523179 \\
\cline { 2 - 4 } & $75 \%$ & 0.0454405 & 0.0038576159 \\
\hline \multirow{7}{*}{100 MBPS } & $35 \%$ & 0.239434 & 0.0054238411 \\
\cline { 2 - 4 } & $50 \%$ & 0.239436 & 0.0050463576 \\
\cline { 2 - 4 } & $75 \%$ & 0.239438 & 0.0046688742 \\
\hline \multirow{2}{*}{1 GBPS } & $35 \%$ & 0.260924 & 0.0031125828 \\
\cline { 2 - 4 } & $50 \%$ & 0.260924 & 0.0031125828 \\
\cline { 2 - 4 } & $75 \%$ & 0.260923 & 0.0041887417 \\
\hline
\end{tabular}

TABLE 4. STATISTICS OF PACKET DELAY VARIATION OVER VARIABLE BANDWIDTH WITH CODEC G.723.1

\begin{tabular}{|c|c|c|c|}
\hline \multirow[t]{2}{*}{ Bandwidth } & \multirow{2}{*}{$\begin{array}{l}\text { Maximum } \\
\text { Reservable } \\
\text { Bandwidth }\end{array}$} & \multicolumn{2}{|c|}{$\begin{array}{l}\text { Packet Delay } \text { Variation } \\
\text { (Sec) }\end{array}$} \\
\hline & & $\begin{array}{l}\text { Non } \\
\text { RSVP }\end{array}$ & RSVP \\
\hline \multirow[t]{3}{*}{$10 \mathrm{Mbps}$} & $35 \%$ & 0.00061168 & 0.000009237 \\
\hline & $50 \%$ & 0.00061168 & 0.0000095364 \\
\hline & $75 \%$ & 0.00061349 & 0.000008841 \\
\hline
\end{tabular}




\begin{tabular}{|l|c|c|c|}
\hline 100Mbps & $35 \%$ & 0.0121124 & 0.000020397 \\
\cline { 2 - 4 } & $50 \%$ & 0.0121122 & 0.000016953 \\
\cline { 2 - 4 } & $75 \%$ & 0.012112 & 0.000016821 \\
\hline \multirow{3}{*}{1 Gbps } & $35 \%$ & 0.0138284 & 0.000010198 \\
\cline { 2 - 4 } & $50 \%$ & 0.0138284 & 0.000010198 \\
\cline { 2 - 4 } & $75 \%$ & 0.0138285 & 0.000013509 \\
\hline
\end{tabular}

\section{$\underline{\text { Result Analysis for Bandwidth } 10 \mathrm{Mbps}}$}

TABLE 5. STATISTICS OF PACKET END TO END DELAY FOR VOIP OVER VARIABLE BANDWIDTH WITH CODEC G.729

\begin{tabular}{|c|c|c|c|}
\hline \multirow[t]{2}{*}{$\begin{array}{c}\text { BANDWIDT } \\
\text { H }\end{array}$} & \multirow{2}{*}{$\begin{array}{c}\text { MAXIMUM } \\
\text { RESERVABLE } \\
\text { BANDWIDTH }\end{array}$} & \multicolumn{2}{|c|}{$\begin{array}{c}\text { PACKET END To End } \\
\text { Delay (SEC) }\end{array}$} \\
\hline & & $\begin{array}{c}\text { NON } \\
\text { RSVP }\end{array}$ & RSVP \\
\hline \multirow[t]{3}{*}{10 MBPS } & $35 \%$ & 0.0673306 & 0.009735099 \\
\hline & $50 \%$ & 0.0673077 & 0.006966887 \\
\hline & $75 \%$ & 0.0673524 & 0.006490066 \\
\hline \multirow[t]{3}{*}{ 100MBPS } & $35 \%$ & 0.0567918 & 0.007284768 \\
\hline & $50 \%$ & 0.056795 & 0.005655629 \\
\hline & $75 \%$ & 0.0567771 & 0.005956953 \\
\hline \multirow[t]{3}{*}{1 GBPS } & $35 \%$ & 0.0705304 & 0.0093046358 \\
\hline & $50 \%$ & 0.0705375 & 0.0067682119 \\
\hline & $75 \%$ & 0.0705269 & 0.0065364238 \\
\hline
\end{tabular}

TABLE 6. Statistics Of Packet Delay Variation Over VARIABLE BANDWIDTH WITH CODEC G.729

\begin{tabular}{|c|c|c|c|}
\hline \multirow[t]{2}{*}{ Bandwidth } & \multirow{2}{*}{$\begin{array}{l}\text { Maximum } \\
\text { Reservable } \\
\text { Bandwidth }\end{array}$} & \multicolumn{2}{|c|}{$\begin{array}{l}\text { Packet Delay } \\
\text { (Sec) }\end{array}$} \\
\hline & & Non RSVP & RSVP \\
\hline \multirow[t]{3}{*}{$10 \mathrm{Mbps}$} & $35 \%$ & 0.00082867 & 0.0000604305 \\
\hline & $50 \%$ & 0.00082916 & 0.0000529801 \\
\hline & $75 \%$ & 0.00083044 & 0.0000521523 \\
\hline \multirow[t]{3}{*}{ 100Mbps } & $35 \%$ & 0.00085107 & 0.000026821 \\
\hline & $50 \%$ & 0.00085105 & 0.000026821 \\
\hline & $75 \%$ & 0.00085098 & 0.000035265 \\
\hline \multirow[t]{3}{*}{1 Gbps } & $35 \%$ & 0.00094984 & 0.000068543 \\
\hline & $50 \%$ & 0.000950252 & 0.000054139 \\
\hline & $75 \%$ & 0.000950538 & 0.000052649 \\
\hline
\end{tabular}

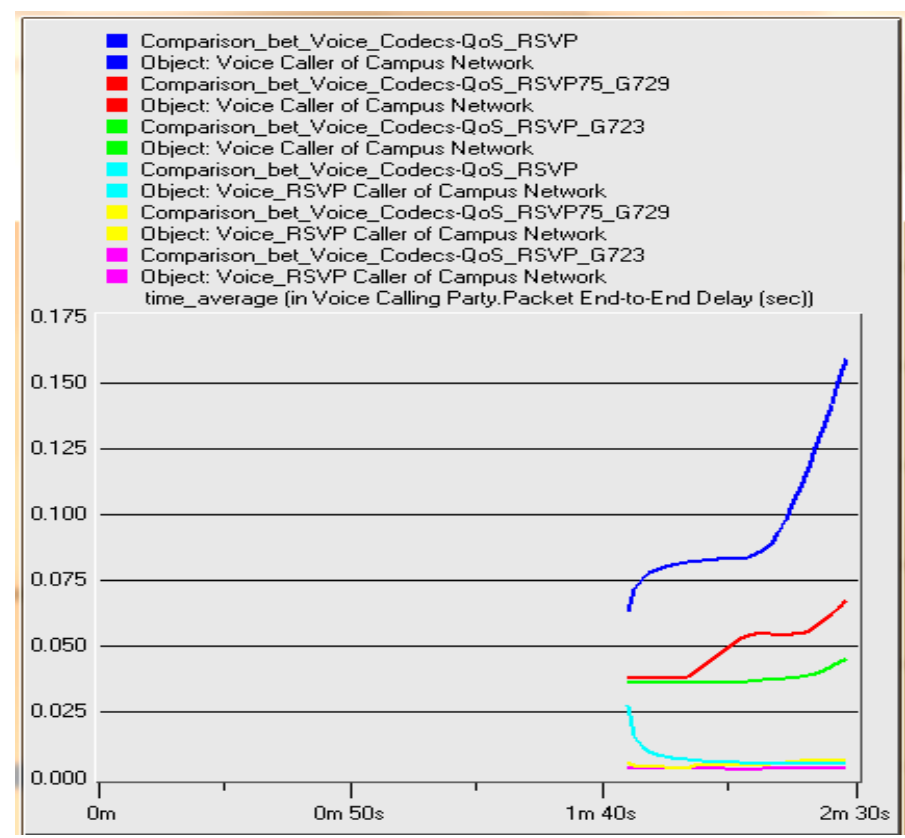

Fig 3. Packet End to End Delay for voice calling at $\mathbf{7 5 \%}$ Reservable bandwidth 


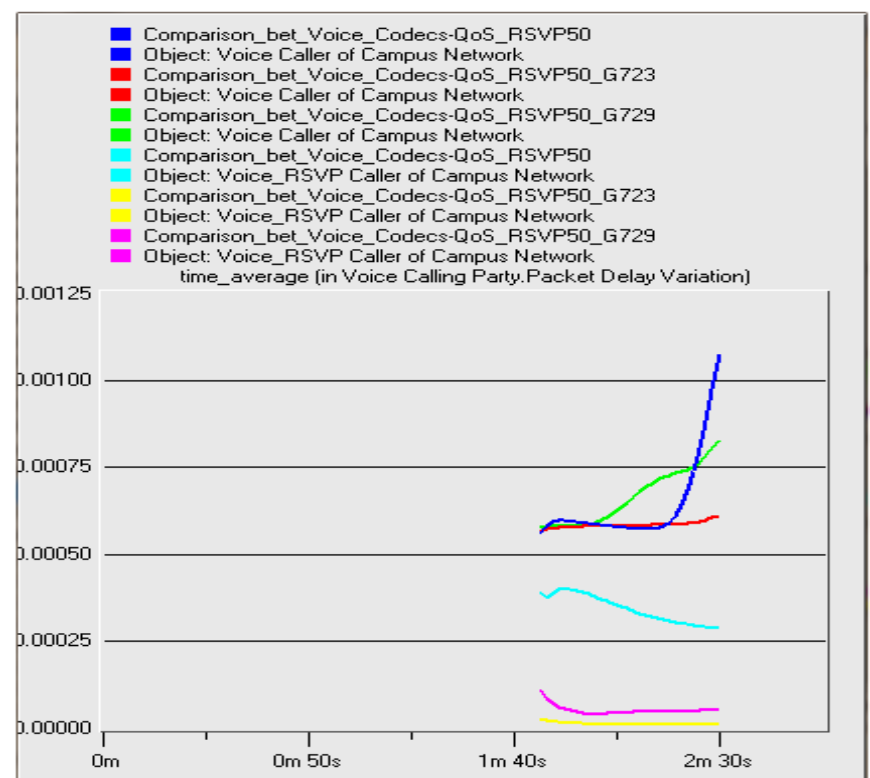

Fig 4. Packet Delay variation for voice calling at $50 \%$ Reservable bandwidth

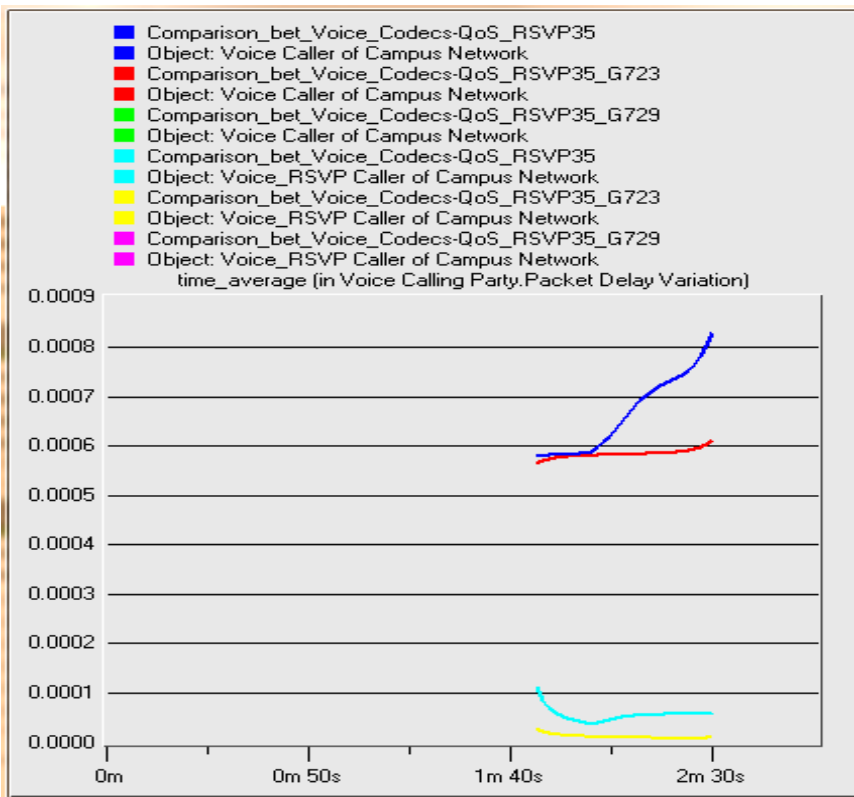

Fig 6. Packet Delay variation for voice calling at $35 \%$ Reservable bandwidth

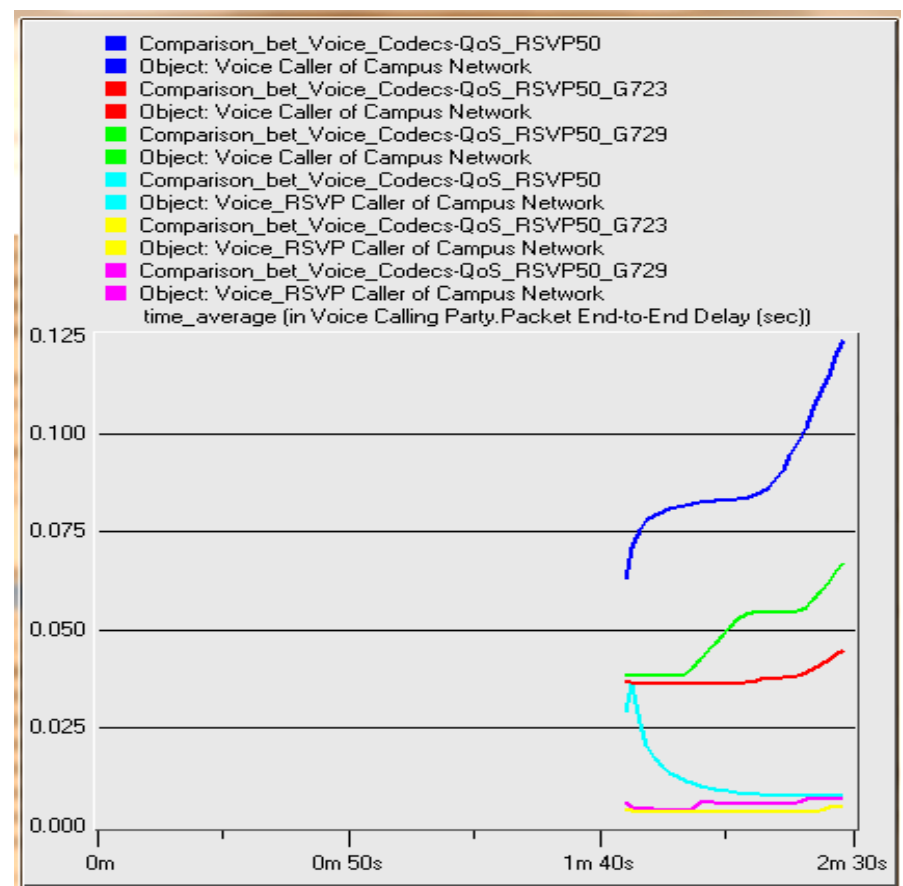

Fig 5. Packet End to End Delay for voice calling at $50 \%$ Reservable bandwidth

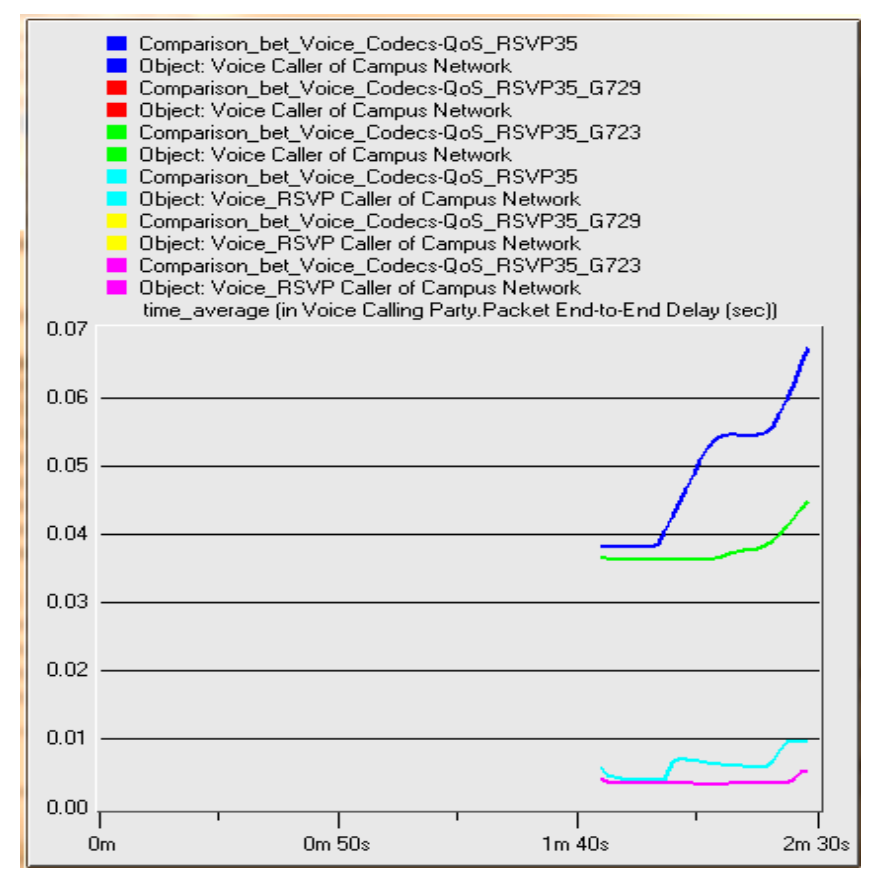

Fig 7. Packet End to End Delay for voice calling at $35 \%$ Reservable bandwidth 
Result Analysis for Bandwidth 100 Mbps

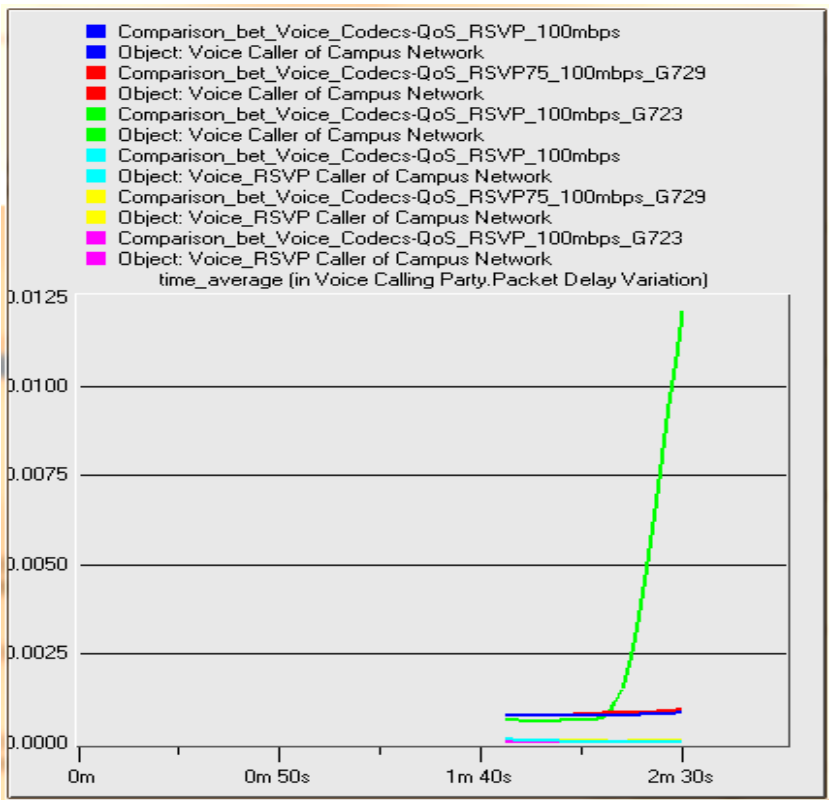

Fig 8. Packet Delay variation for voice calling at $75 \%$ Reservable bandwidth

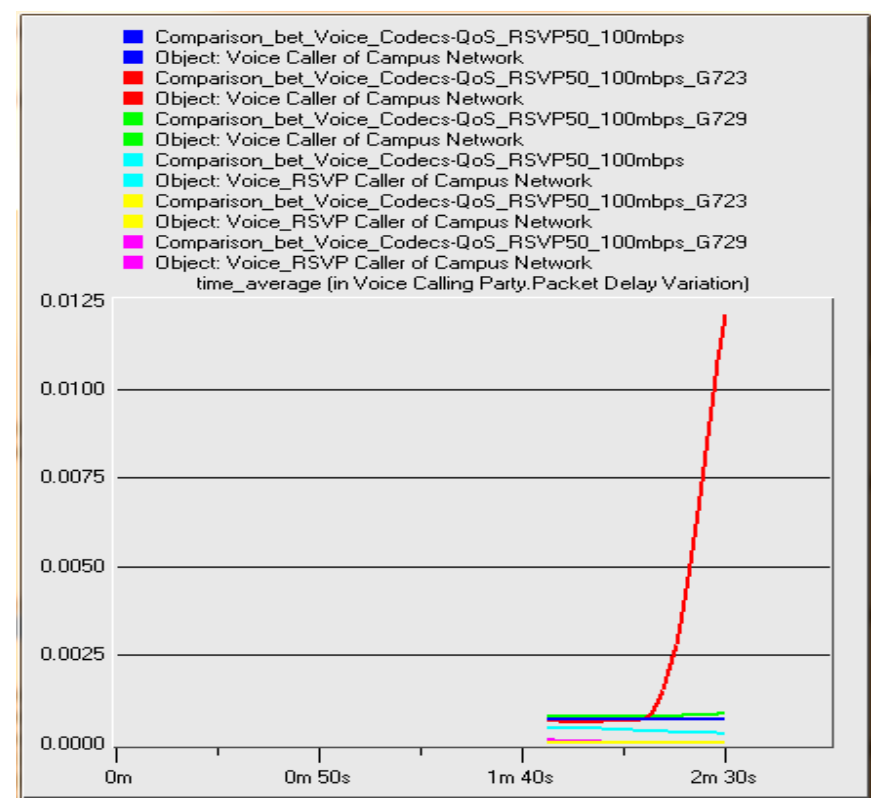

Fig 10. Packet Delay variation for voice calling at $50 \%$ Reservable bandwidth

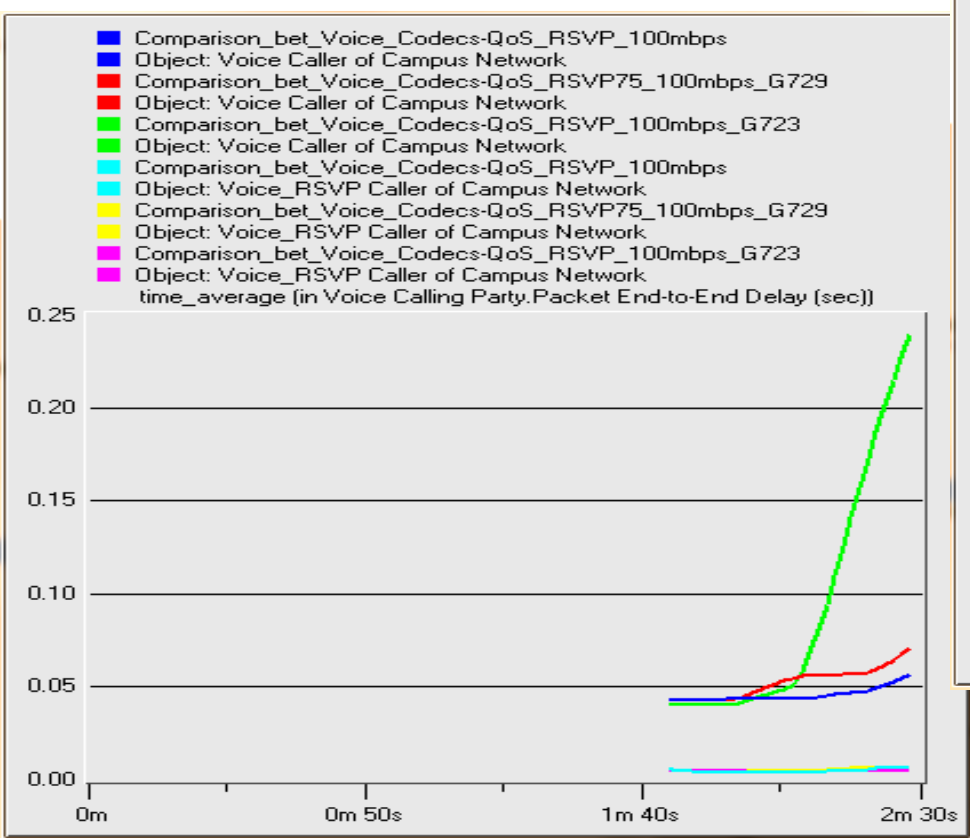

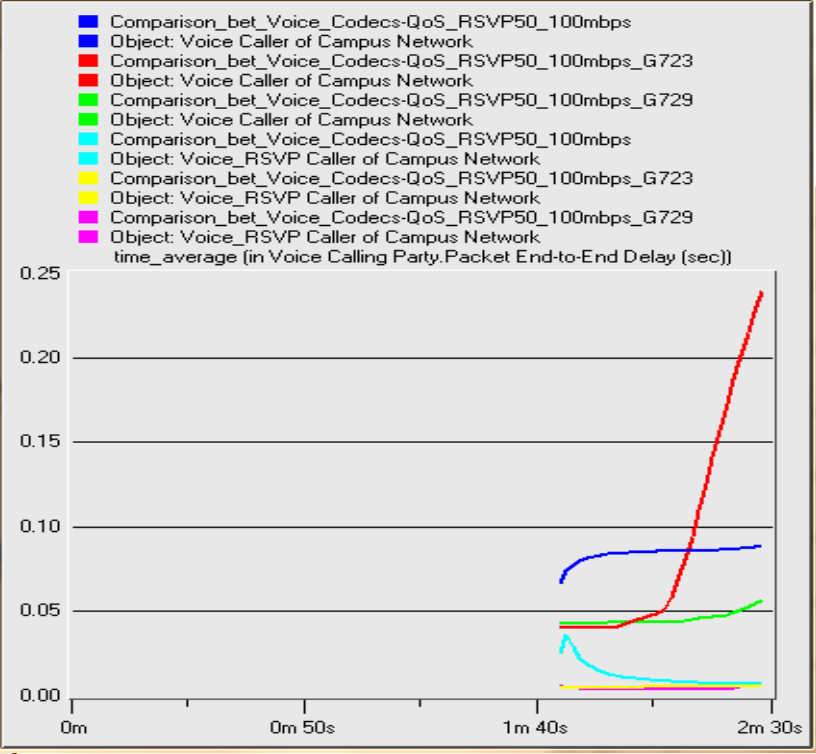

Fig 11. Packet End To End for voice calling at $50 \%$ Reservable bandwidth

Fig 9. Packet End To End for voice calling at $75 \%$ Reservable bandwidth 


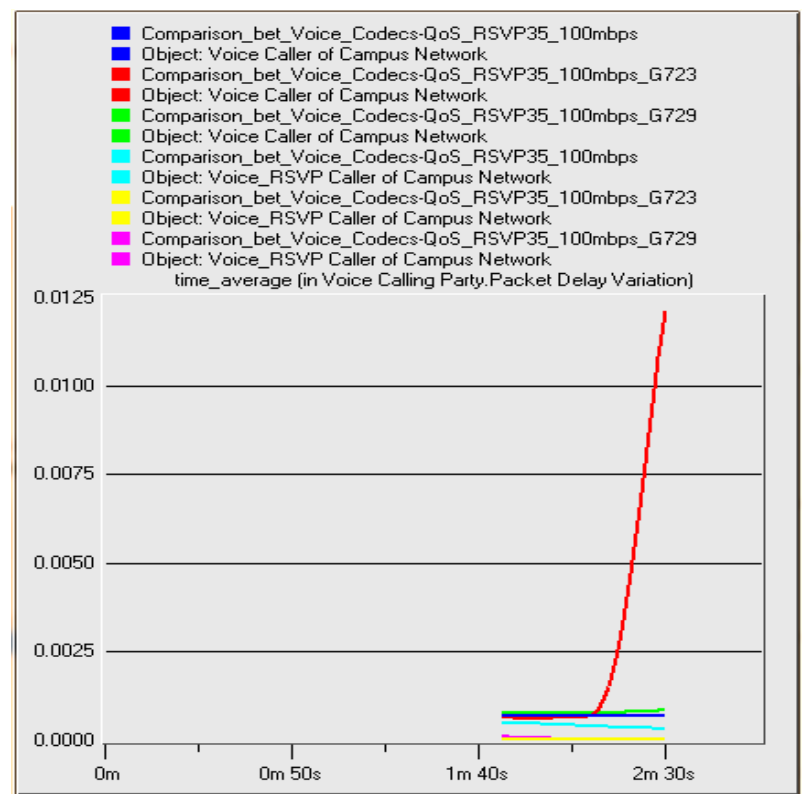

Fig 12. Packet Delay variation for voice calling at $35 \%$ Reservable bandwidth

\section{Result Analysis for Bandwidth 1Gbps}

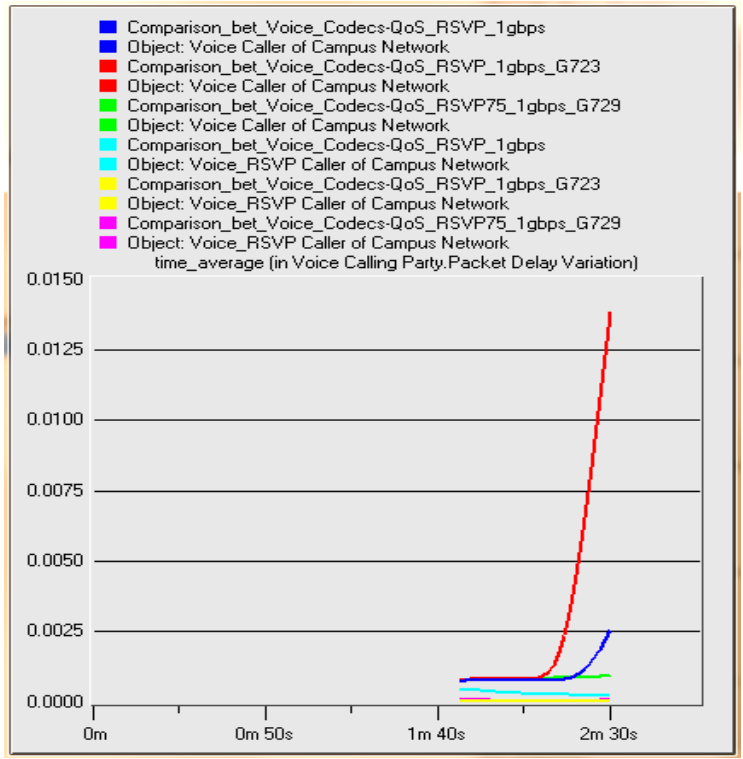

Fig 14. Packet Delay variation for voice calling at $75 \%$ Reservable bandwidth

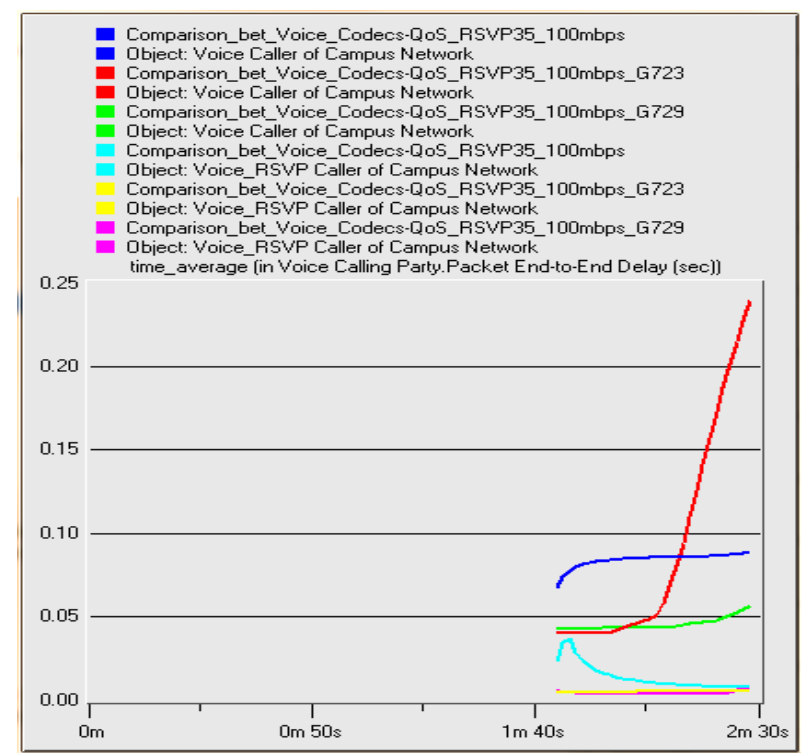

Fig 13. Packet End To End for voice calling at $35 \%$ Reservable bandwidth

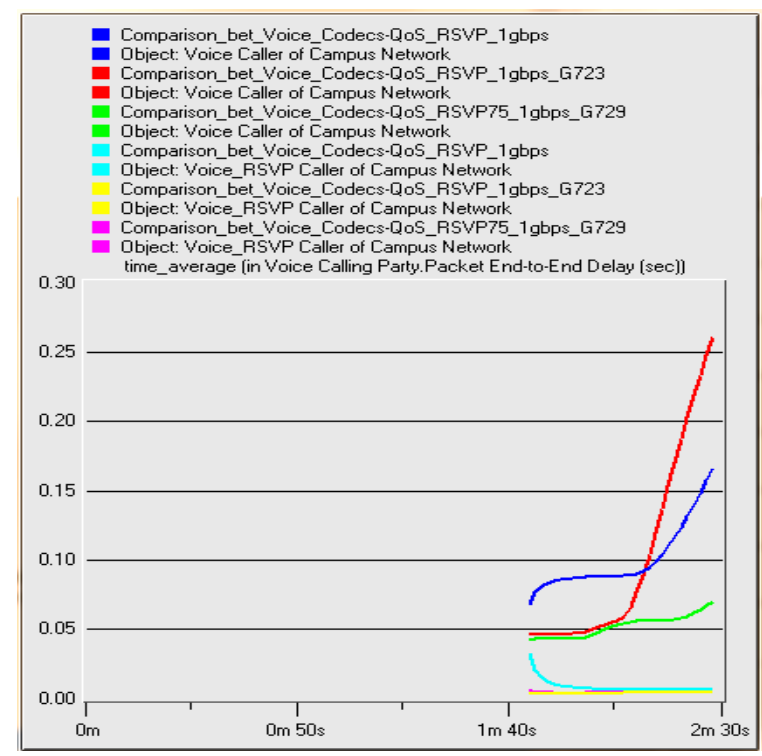

Fig 15. Packet End To End for voice calling at $75 \%$ Reservable bandwidth 


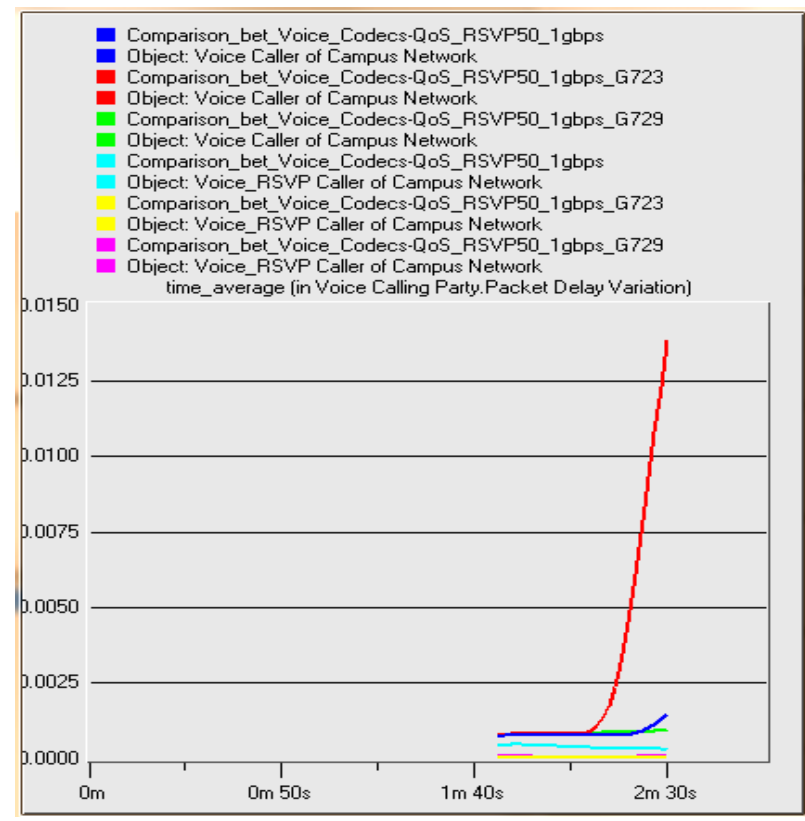

Fig 16. Packet Delay variation for voice calling at $50 \%$ Reservable bandwidth

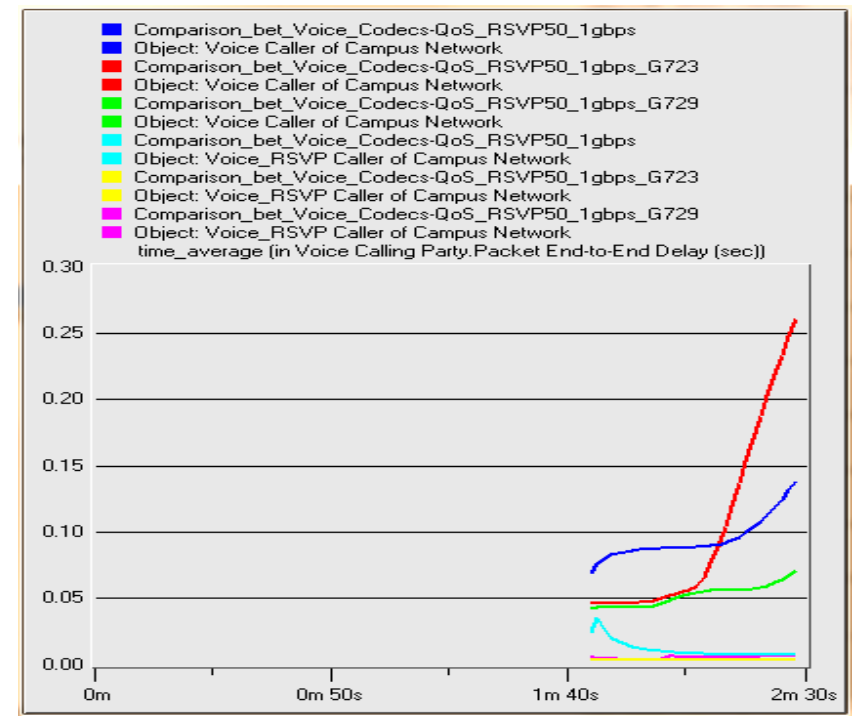

Fig 17. Packet End To End for voice calling at $50 \%$ Reservable bandwidth

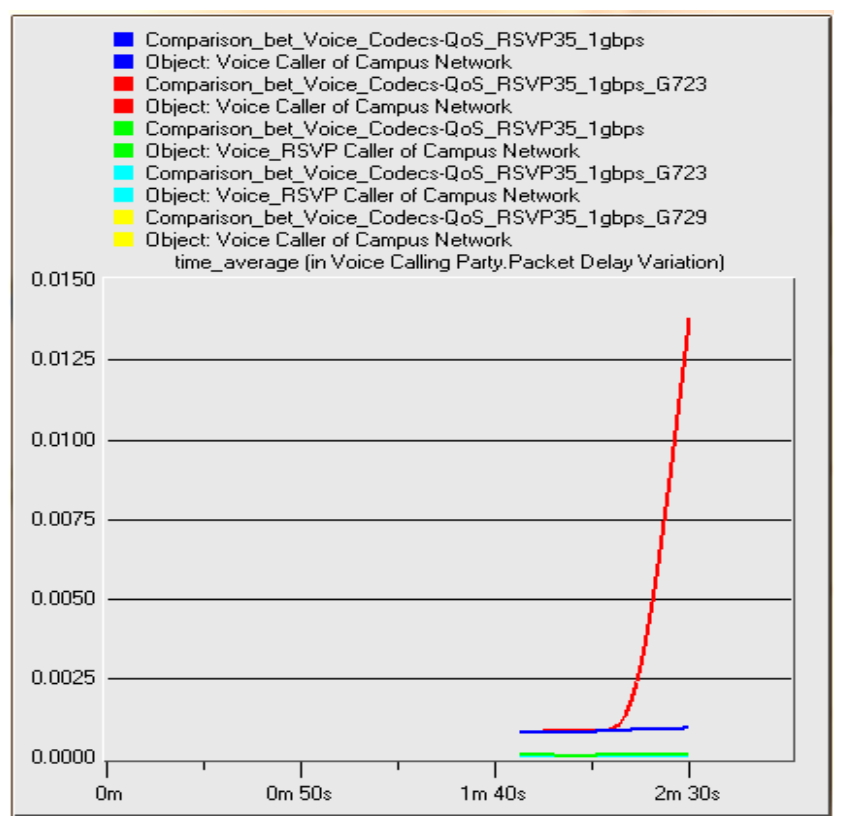

Fig 18. Packet Delay variation for voice calling at $35 \%$ Reservable bandwidth

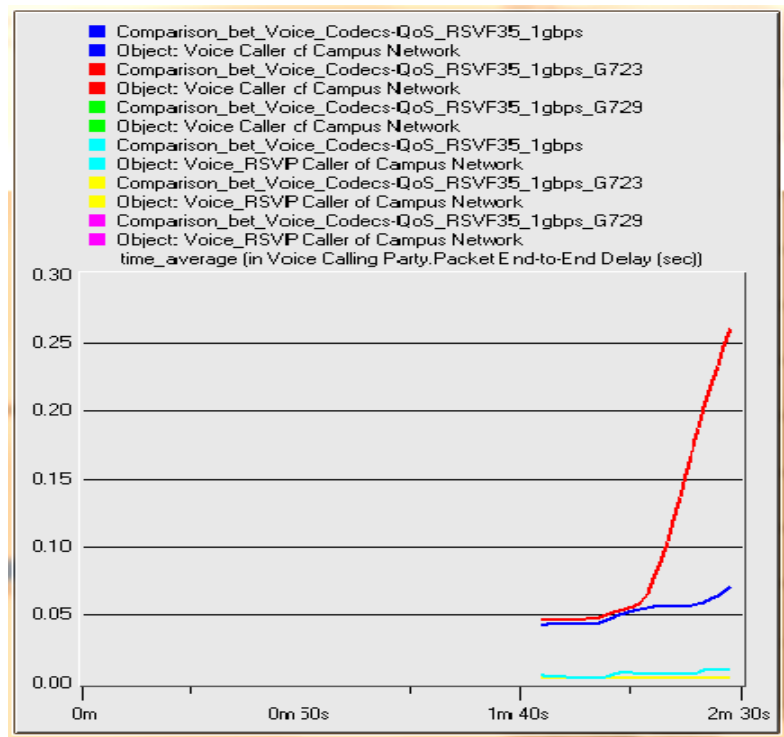

Fig 19. Packet End To End for voice calling at $35 \%$ Reservable bandwidth

Fig 2 to Fig 7 shows packet delay variation and end to end delay for voice traffic for RSVP and non RSVP network for $10 \mathrm{Mbps}$. In both the cases end to end delay and packet delay variation is considerably low for RSVP network when CODEC G.723.1 is used.

Fig 8 to Fig 13 shows packet end to end delay for voice calling for RSVP and non RSVP network for 100Mbps. For RSVP, packet end to end delay and packet delay variation is less when used with 
CODEC G.723.1. But for Non RSVP application, end to end delay and delay variation is less when G.729 is used.

Fig 14 to Fig 19 shows packet end to end delay for voice calling for RSVP and non RSVP network for 1Gbps. Here also for RSVP, packet end to end delay and packet delay variation is considerably less with CODEC G.723.1. For Non RSVP application, end to end delay and delay variation is less with CODEC G.729.

\section{CONCLUSION}

Packet end to end delay and packet delay variations are the two major parameter that helps to determine the quality of voice. If both are less, then the quality of service is good. One of the unexpected results of the RSVP research effort has been the realization that Packet end to end delay and Packet delay variation are lesser for RSVP enabled client as compared to Non RSVP Client. Here the network architecture has been tested on variable bandwidth condition for voice based application. Results have been evaluated for both non RSVP and RSVP network. It has been observed that, for a given bottleneck link capacity, as the requirement of voice application increases, Packet end to end delay and Packet delay variation for RSVP enabled client decreases as more bandwidth is allocated to RSVP client. On other side, Packet end to end delay and Packet delay variation for Non RSVP client increases as less bandwidth is available to transfer their packets. RSVP's receiver-initiated reservations accommodate heterogeneous QoS requests from different receivers. It has been observed that G.711 codec has the highest Packet end to end delay and Packet delay variation, on the other hand, G.723.1 has the lowest Packet end to end delay and Packet delay variation .It shows fair results from $10 \mathrm{Mbps}$ to $1 \mathrm{Gbps}$ which reflects that it can be used in $3 \mathrm{G}$ as well as $4 \mathrm{G}$ networks.

Hence by using RSVP Protocol in combination with G.723.1 codec gives better performance comparing with other Codec and general objective can be achieved by using G.723.1 codec. Results are useful for QoS support for Next Generation Network.

\section{ACKNOWLEDGMENT}

Our sincere thanks to Thakur Educational Trust and Management to provide all the facilities and infrastructure to carried out the research work.

\section{REFERENCES}

[1] Costa, Nelson, Nunes, Má, rio Serafim, "Adaptive Quality of Service in Voice over IP Communications, "Fifth International Conference on Networking and Services, 2009. ICNS '09, Valencia, Spain, 20-25 April 2009, pp. 19-24.

[2] C. Shen, W. Seah, et al., Mobility Extensions to RSVP in an RSVP-Mobile IPv6 Framework, Internet draft (July 2002) [Online]

[3] Eunkyu lee,Sang Ick Byun, Myungchul kim “A Translator between Integrated services RSVP and differentiated services for End to End QOS”. IEEE March 2003 Page(s): 1394 1401 vol.2.

[4] G. Camarillo, W. Marshall, J. Rosenberg, Integration of resource management and SIP, IETF RFC 3312, October, 2002.

[5] ITU-T Recommendation G.114: One-way transmission time; 05/2000.

[6] ITU-T Recommendation G.711: Pulse code modulation (pcm) of voice frequencies; 11/1988.
[7] ITU-T Recommendation G.729: Coding of speech at $8 \mathrm{kbit} / \mathrm{s}$ using conjugate-structure algebraic-code-excited linearprediction (CSACELP); 03/1996.

[8] ITU-T Recommendation G.726: 40, 32, 24, 16 kbit/s adaptive differential pulse code modulation (ADPCM); 12/1990.

[9] ITU-T Recommendation G.723.1 : Dual rate speech coder

for multimedia communications transmitting at 5.3 and 6.3 kbit/s; 03/1996.

[10] ITU-T Recommendation G.113: Transmission impairments due to speech processing; 02/2001.

[11] Jonathan Davidson, Voice over IP Fundamental (2nd Edition), Cisco Press, 2006.

[12] J. Manner, K. Raatikainen, Localized QoS Management for Multimedia Applications in Wireless Access Networks, in: Proc. IASTED IMSA 2003 (January 2003).

[13] Mubashar Mushtaq and Toufiq ahmed "Multimedia Caching Integration for Efficient content Delivery over Heterogeneous Networks". March 31 2008-April 42008 on page(s): 1011-1012.

[14] Nen chaung Wang and jyh-Woei Jiang "Extending RSVP for QOS support in Heterogeneous wireless networks".ICCS 2006. 10th IEEE Singapore International Conference. Volume, Oct. 2006 Page(s): 1 - 5.

[15] Neill Weilkinson "Next Generation Network Services Technologies and Strategies" john willey and sons ltd pp 167-170.

[16] Pascal Lorenz, "QOS in Next Generation Network. $26^{\text {th }}$ International Conference Interfaces ITI 2004, June 7-10.

[17] S.Cherry, "Seven Myths About Voice over IP", IEE Spectrum, March 2005.

[18] Shahrizal Sahabudin , Mohamad Yusoff Alias "End-to End Delay Performance Analysis of Various Codecs on VoIP Quality of Service" IEEE Proceedings 2009 Page(s):607-612

[19] Talukdar, B. Badrinath and A. Acharya, MRSVP: a Resource Reservation Protocol for an Integrated Services Network with Mobile Hosts, Wireless Networks 2001.

[20] Tomas Robles, Hector velyous, Arndt kadelka, "QOS for all IP System beyond 3g", Communications Magazine, IEEE Volume 39, Issue 8, Aug 2001 Page(s):64 - 72

[21] Yorem Benet "The complementary role of RSVP and differentiated services in the full service QOS network." Communication Magazine IEEE Feb 2000. Volume:38, On page(s): 154-162.

[22] $\mathrm{Yu} \mathrm{Ge}$, Winston seah "A method to efficiently integrate Internet Telephony calls signaling with dynamic resource negotiation. Computer Networks Vol 50, Issue 17 Pages 3334-3352 